\title{
BREAKING: Password Entry is Fine
}

\author{
Catie Jo Pidel and Stephan Neuhaus \\ Zurich University of Applied Sciences \\ Zurich, Switzerland \\ \{catlin.pidel, stephan. neuhaus\} @zhaw.ch
}

\begin{abstract}
In our digital world, we have become well acquainted with the login form-username shown as plaintext, password shown as asterisks or dots. This design dates back to the early days of terminal computing, and despite huge changes in nearly every other area, the humble login form remains largely untouched. When coupled with the ubiquity of smartphones, this means we often find ourselves entering complex passwords on a tiny touchscreen keyboard with little or no visual feedback on what has been typed. This paper explores how password masking on mobile devices affects the error rate for password entry. We created an app where users entered selected passwords into masked and unmasked password fields, measuring things like typing speed, error rate, and number of backspaces. We then did an exploratory data analysis fo the data, and our findings show that, perhaps unexpectedly, there is no significant difference between masked and unmasked passwords for any of these metrics.
\end{abstract}

Keywords: security $\cdot$ passwords $\cdot$ data entry errors $\cdot$ mobile security

\section{Introduction}

The average person logs into 7-25 accounts every day [4], and the vast majority of people memorize their passwords, meaning reuse of identical or similar passwords is common [13]. This stands in opposition to traditional security advice, which dictates that passwords should be long, random strings full of complex characters that are changed regularly and never reused across accounts. This can really only be done by using a password manager, which only $3 \%$ of Americans use consistently [13]. What is more, in the smartphone era, tiny keyboards with separate screens for letters and special characters can turn a strong password into a usability nightmare: mobile users mistype their passwords twice as often as desktop users, and it also takes $20 \%$ longer on average for them to do so [12].

Password unmasking is the practice of displaying passwords in plaintext rather than the traditional censored format. Is this a viable alternative? Usability experts claim that masking passwords is an unnecessary complication that causes confusion and frustration. But some security experts warn against the dangers of shoulder surfing (stealing passwords by looking over people's shoulders as they type). While there are a number of articles and blog posts espousing one view or the other, there is little hard evidence on how much password masking actually hinders usability. This paper aims to take on a small part of this overlying issue: how does the error rate for password entry compare between masked and unmasked passwords, specifically for smartphone users? 


\subsection{To Mask or Not To Mask}

Password masking comes from terminal computing, when computers were mostly used by scientists, and every command was printed out on paper [9]. At that time, it made sense not to echo passwords at all or at least to replace them with asterisks; without it, anyone with access to the computer printouts could easily harvest someone else's login info. But this rationale no longer applies. The days of needing to be an expert to use a computer are long gone, and there is no printout to worry about. Still, users and security experts alike balk at the idea of showing passwords in plaintext. What if someone looks over your shoulder without you knowing? Additionally, how do you navigate the trust dynamics of entering a password with someone else present? Is it alright to ask your boss, your partner, or your child to look away [19]? From a usability perspective, password masking also helps communicate that a password is sensitive information [19]. It is also what the user expects-a recent study on password unmasking showed that $60 \%$ of users grew suspicious when their passwords were unmasked by default [11]. Even though masking is a purely cosmetic fix, removing it tends to make users think there is a bug or even a security threat on the site in question [11].

Some prominent usability experts point out that the lack of visual feedback flies in the face of usability guidelines $[19,15]$ : an error-prone login experience frustrates users, and can even be a security threat. Rather than risking having to retype a complex password, users opt for simpler versions, or even for copy-pasting from a text file [19, 15, 14]. Additionally, it is much harder to snoop a password typed onto a smartphone screen, and with mobile Internet usage recently overtaking desktop traffic [4], the threat of shoulder surfing is becoming less common relative to the nuisance of repeatedly typing a password [20].

Despite having been pronounced dead many times [9], passwords are the most used authentication mechanism on the Internet today [10]. Similarly, mobile phones are not disappearing in the immediate future, so gaining a deeper understanding of entering a password on a mobile device is a relevant issue. For as long as passwords have been around, we are still in a "data poor" research state [10], so this study contributes to password research as well as the growing intersection between usability and security research.

\subsection{Contributions}

We specifically make the following contributions:

- We compare the error rates of different types of password on mobile phones.

- We tested two different types of login screens: the standard, masked password, and a completely unmasked password.

- Additionally, we tested these logins with different types of passwords to see what role the password length and density of special characters play in this. 


\section{Related Work}

\subsection{Usability of Password Masking}

There has been considerable debate over the usability of masked passwords. Usability expert Jakob Nielsen [15] and security expert Bruce Schneier [19] have argued that masking passwords causes users to choose easier (and therefore less secure) passwords, or equally problematic, copy and paste them from a file. They downplayed the threat shoulder surfing, a point which many of the commenters on these articles disagreed with. They argued that using a computer in a public setting like a coffee shop, as well as needing to enter a password during a presentation, are legitimate use cases for password masking. Additionally, a small-scale user study also showed that unmasking passwords broke users' expectations and even undermined their trust- $60 \%$ of participants became suspicious of the site when their passwords were displayed in plaintext [11].

Despite the considerable debate, we were unable to find research directly comparing the error rate between masked and unmasked password fields. How much of a difference does an unmasked password really make?

\subsection{Usability And Security of Different Types of Logins}

There is also a fair amount of research into alternatives for the username and password combination, such as the usability of click-based graphical passwords [2] and password managers [3]. One study generated a "profile" of how the user types their password, based off of the time a key is held down and the time between key presses [17]. When the profiles of valid users were compared against those of impostors entering the same password, this additional metric helped filter out non- authorized users. However, typos and the subsequent use of backspace interfere with this metric, making it not feasible for large-scale adoption. Another study explored an alternative to password masking, the TransparentMask [8]. This combines the typical black dots with symbols that represent a hash of the last $\mathrm{n}$ characters of a password. The idea is that since humans can easily recognize sequences of symbols, it would provide a way to alert the user to typos without providing as much of a security risk as a fully unmasked password.

Transparent Mask example [8]

While these ideas are fascinating, their likelihood for widespread adoption is unlikely, and may also lead to user confusion. According to one survey of web tools [14], login forms are among the least standardized website components. Introducing new paradigms, however well-intentioned, may only serve to muddy the waters. Additionally, while the typical login form is not as interesting, its persistence in our digital world demands more research than has currently been done on the subject $[1,10]$.

\subsection{Mobile Password Entry}

As mobile phone usage continues to increase [4], passwords are increasingly being entered on mobile devices. And while mobile devices are often cited as a reason why password unmasking is important, we did not find studies measuring how much masked 
password fields and tiny keyboards affect usability. One dissertation provides an indepth analysis of password entry on mobile devices, what influences the user typing speed (such as switching keyboards to find special characters), and the types of mistakes commonly made when entering a password [5]. Prior research has also explored the error rate of typing passwords on mobile phones compared to desktops [12], as well as an analysis of the "number and nature of errors committed during password entry" and whether the user notices these errors before submitting the password [7]. Our work provides another facet to the question of mobile password entry by studying the error rates for both masked and unmasked login prompts.

Schaub et al. [18] examined how the design of different smartphone keyboards (iOS, Windows, Android, and others) can make shoulder surfing easier or more difficult. There has also been research into how the platform (mobile, tablet, desktop) affects the makeup of the password created [21], but not whether certain types of passwords are more or less error-prone in daily mobile use.

\section{Methodology}

This study aims to answer two questions. The first is how does password masking affect the error rate of password entry on mobile devices? Secondly, how does the makeup of of a password influence the aforementioned error rate?

\subsection{Effects of Password Masking}

We tested two different login forms - one with typical mobile password masking (where the password is masked except for the most recently typed character), and a fully unmasked password, displayed in plaintext. We also considered testing a third option where the user has the choice to mask or unmask their password with a checkbox, but we ultimately decided that this was better tested in a separate study concerned with whether users will choose to change the default masking of a password.

\subsection{Effects of Password Makeup}

There is much variation in passwords, but we have identified four main categories:

- Pass phrases: multiple words separated by spaces ("cats are fantastic friends")

- Typical passwords: a single (in our case, English) word with letters replaced by numbers and special characters ("C@terp! 11ar2018").

- Randomized: a fully randomized string of characters, such as that used by a password manager ("nBqzEcP $2 A\}$ Q $8, j G$ ")

- Bad passwords: passwords often seen in password leaks ("password123")

Of these four types, we are not interested in "bad passwords" because they have already been shown to be a security threat. For the 3 more secure alternatives, we generated a list of 10 passwords at equivalent password strength (as measured by our chosen software, 1Password). Our hypothesis is that the error rate for password entry varies both by type of password and by whether the password field is masked. 


\subsection{Study Structure}

Our study involves the use of a custom-built app, PasswordResearcher, for user testing. This app is built in Unity and deployed to both iPhone and Android, and is available with either English or German keyboards depending on the preferences of the user. This is to mitigate any errors that could arise from using an unfamiliar keyboard layout.

Each session has two parts: one half where the passwords are masked and the other with passwords unmasked. We alternate whether the participant starts with the masked or unmasked task each time. To ensure there was no overlap of passwords between these two portions, the app randomly chooses four passwords from each of the three categories and splits them into two groups of six passwords (two from each category). For each half of the study, the participant sees a given password three times, appearing in a random order. Entering the password multiple times is intended to elicit a learning response since participants learn to type their passwords more quickly and consistently over time. Additionally, the random shuffling is to allow us to test all three password types under similar conditions-it controls for participants getting bored and therefore less careful over time, or alternatively, getting more used to typing and getting better.

The passwords are displayed on the screen as an image so it is not possible to copy-paste the on-screen password, and also so the user does not need to memorize the password. We did not lie to our participants for ethical reasons, but we were also careful not to mention that we were interested in the error rate of password entry since this could change their natural behavior and skew our results.

\subsection{Data Collected}

We used a recruiting agency, TestingTime ${ }^{1}$, to recruit ten participants. For each password attempt, we recorded:

- The participant ID

- The expected (correct) password

- The actual password typed

- The time taken to enter the password

- The type of password (pass phrase, random, or typical password)

- Overall attempt number (1 to 18 for each half)

- Password attempt number (1 to 3)

- Whether attempt is done on Android or iOS

It is worth noting that this study is only concerning itself with whether a login attempt would be successful or not, so we are only interested in measuring errors on submission rather than incremental errors made while typing. The latter has potential for a follow-up study, but is outside the scope of this one.

We also collected qualitative exit questions from each participant:

- Do you remember any of the passwords you typed?

- Which type of the password types, if any, did you feel were quicker to type?

- Which of these password types, if any, did you feel were easier or more difficult to type?

${ }^{1}$ https://www.testingtime.com/en/ 


\section{Results and Analysis}

We defined an incorrect password attempt as one where the actual password entered by the user does not match the expected password. We used the Levenshtein edit distance to further quantify the correctness of a password and to give a lower bound on the actual number of mistakes that were made by the user.

After collecting the data, we removed any attempts that took more than $100 \mathrm{sec}$ onds. This happened occasionally when the participant set down the phone to come ask a question. Similarly, we removed any attempts with more than ten mistakes. These sometimes happened when participants accidentally pressed the enter key too soon.

Because of the small sample size and study constraints, we opted to explore and visualize results without trying to prove statistical significance (see Section 6). The conclusions below are our subjective interpretation of the data.

\subsection{Password Masking versus Error Rate}

Going into this study, we expected to see a lower error rate on unmasked passwords. This made logical sense-participants can visually inspect an unmasked password for errors, whereas masked passwords seemed more prone to "fat finger" mistakes (touching an adjacent key without realizing it). So we computed the raw error rates, which we defined as the total number of incorrect attempts (where the expected and actual passwords are not the same) divided by the total number of attempts. Unmasked passwords did indeed have a lower error rate, but not nearly as dramatically as we would have expected. The average error rate for all types of passowrd entry was $16 \%$, split into $18 \%$ for masked entry and $15 \%$ for unmasked entry.

We then started plotting the data, looking for visual indications of a difference between masked and unmasked passwords. Perhaps unmasked passwords were quicker to type? After all, the participant could visually inspect the unmasked password for errors after typing it.

There was definitely a pretty broad range in the time it took to enter a password, but masked and unmasked data points are nearly perfectly interleaved. We next compared the overall entry time for masked and unmasked passwords (Figure 2, below), and while there was a difference, it was quite slight.

\subsection{Device Type versus Error Rate}

It seemed clear that the difference between the error rates for masked and unmasked passwords was very small, so we started looking for other interesting features of the data. We had participants using both iPhone and Android devices - was one platform easier to use and therefore less error prone? (See Figure 3.)

Even just visually inspecting the plots, it was hard to see a difference between the Android and iOS columns. So we went back to the drawing board. 


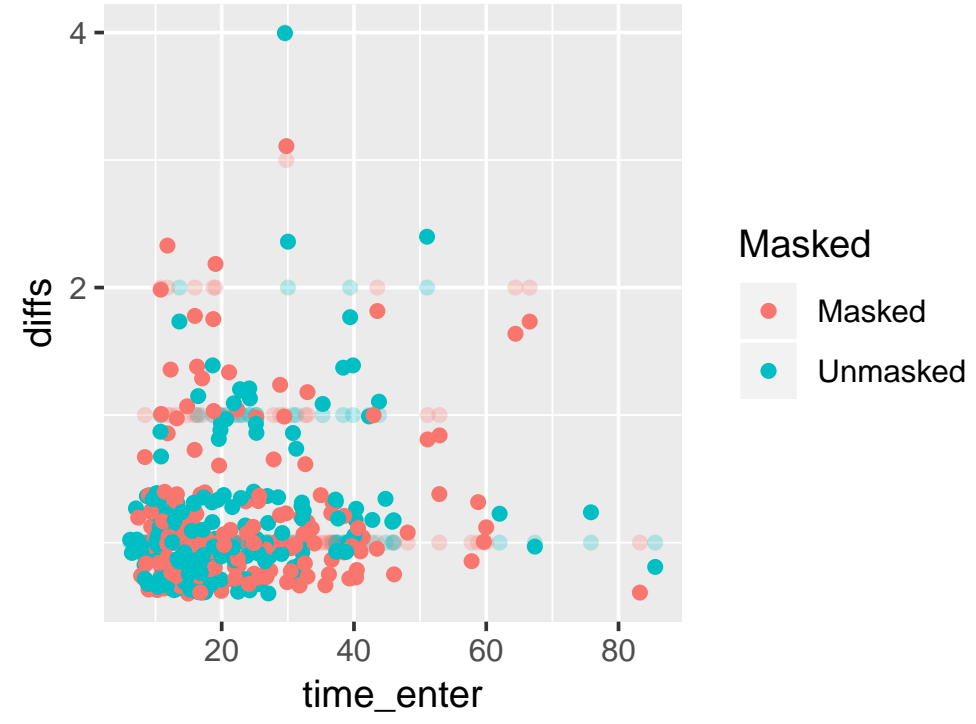

Fig. 1. Time to enter a password versus the number of differences between the expected and actual password. Jitter is added to the points in order to show the clustering of the data, but all differences are integer-valued.

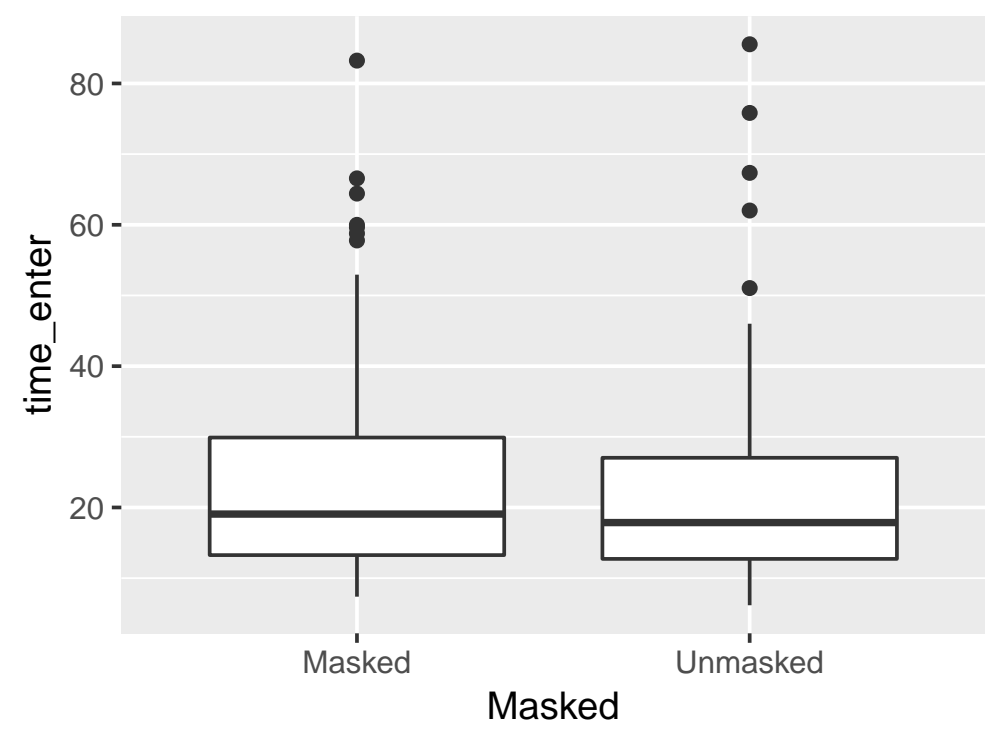

Fig. 2. Boxplot of each the time it took to enter passwords with each password masking. 


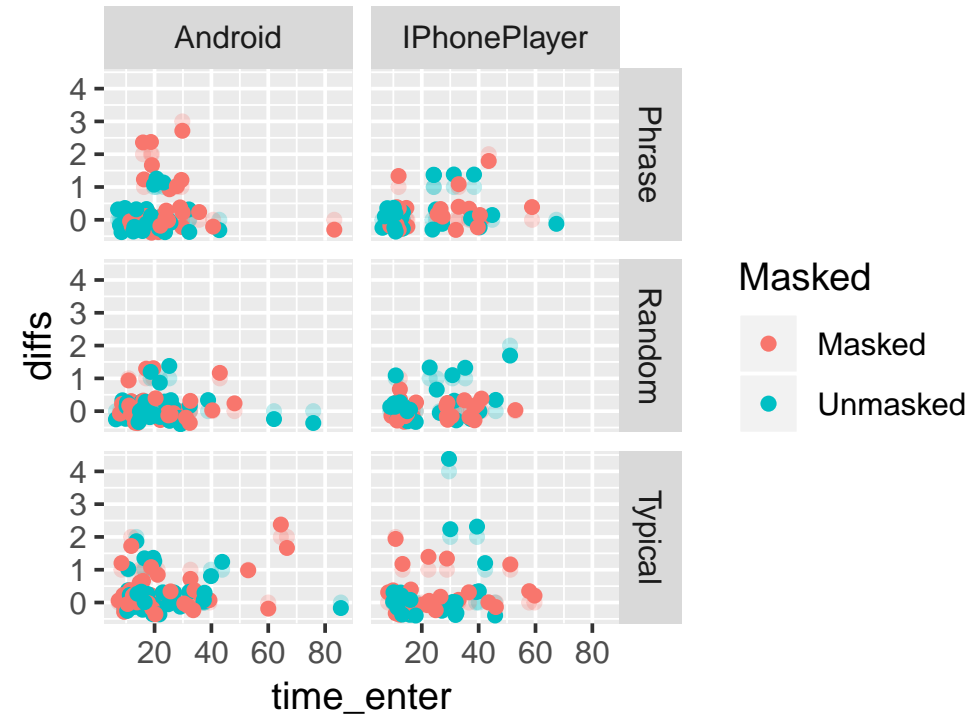

Fig. 3. Number of mistakes versus entry time, split by OS and password type. Points are jittered, $y$ values are integers.

\subsection{Password Type versus Error Rate}

Neither password masking nor device type affected the error rate more than is expected by chance. Then we wondered about each of the password types - since a random password is much shorter than a multi-word password, we decided to compare the masked and unmasked entry times per password type. Given that all the passwords chosen for the study were equivalently secure, was there a certain type of password was more error prone? We plotted the same graph as shown in Figure 1, this time coloring the points by the password type instead of whether it was masked or unmasked. (See Figure 4.)

Just looking at the graph, it was clear that there was once again no real difference, so we turned ourselves towards one last metric: was a certain password type faster to type than the others? (See Figure 5.)

Answer: not really. Even comparing each participant's typing speeds, the graphs for each password were more or less the same. This is actually interesting, given the difference in length between an 11-character random password and an upwards-of- 20character multi-word password. This also aligns with similar research, which shows random passwords with special characters took considerably longer to type than standard text of the same length [5]. As for why this occurs, our qualitative results were in agreement with the previous work: users have to spend considerable time hunting for special characters.

\subsection{Qualitative Analysis}

Following each user session, we asked the same three exit questions. 


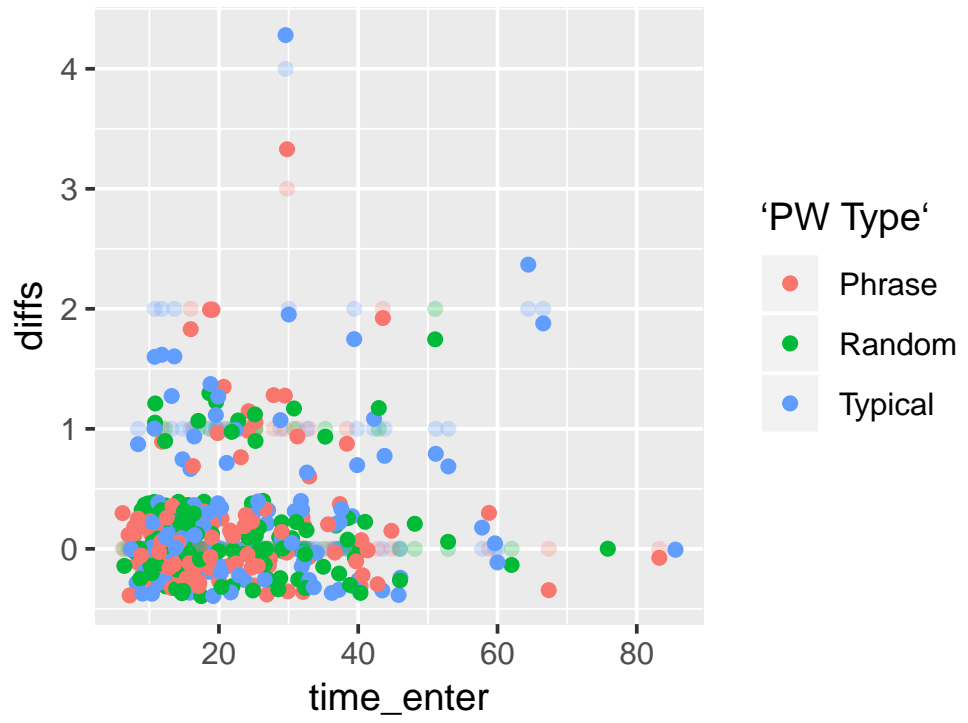

Fig. 4. Number of mistakes versus entry time, split by password type. Points are jittered, $y$ values are integers.

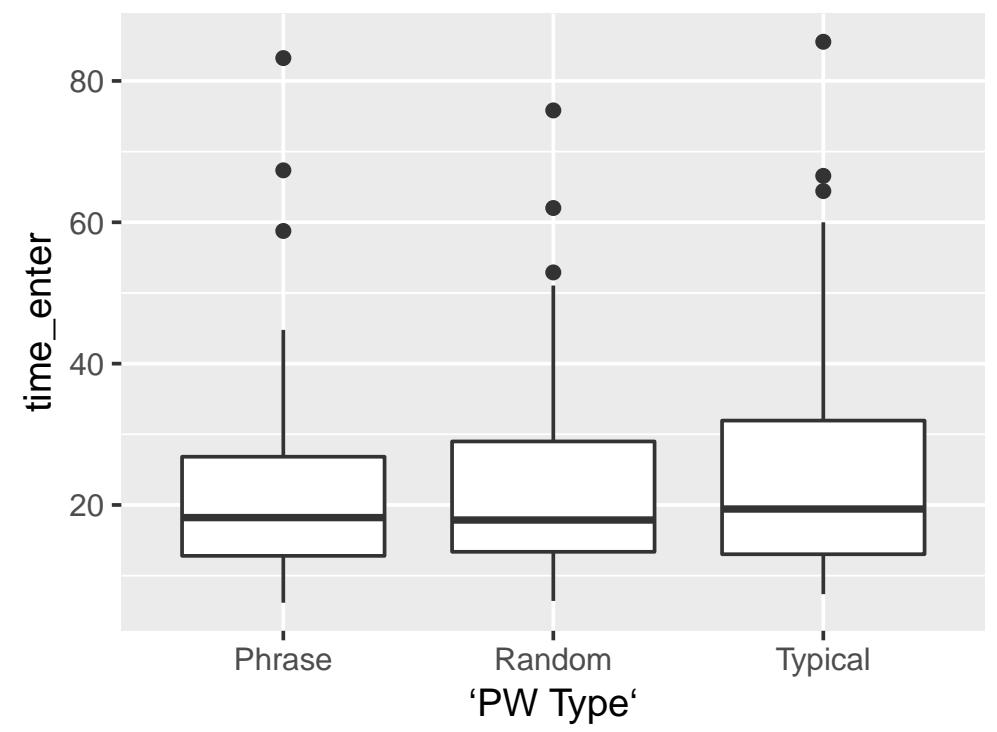

Fig. 5. Entry time versus password type. 
Do you remember any of the passwords you typed? One participant could name nearly half the passwords, even some random ones, letter for letter. Another could not name a single thing they typed. The random passwords were definitely the least remembered, and while people could usually remember parts of the other two types, there were frequent mistakes. For example, they would confuse the order of words in a multiword password, or mix the words up between multiword passwords. For more typical passwords, they often remembered words, but would make mistakes on the exact placement of special characters, such as confusing "Cr@ck3rJacked" as "Cr@ckerJack 3 d".

Which of the password types, if any, did you feel were quicker to type? Nearly everyone said that the multiword passwords were easiest to type. We were surprised, however, that several participants also added, unprompted, that they would not choose such passwords since the lack of special characters and numbers made them less secure.

Which of these password types, if any, did you feel were easier or more difficult to type? For this question, we showed them a list of the passwords they had typed for the study, and asked them to point out any passwords that were particularly easy or difficult, and why. There was one password that was significantly longer than the other passwords, "jubilant wineshop sceptic cadenza", which was nearly always pointed out as difficult when participants encountered it. They would also point out characters they found ambiguous (such as a lower case 'l' and the upper case vowel, 'I').

\section{Discussion}

We observed a $2.1 \%$ higher error rate when passwords are masked. Based on the study size, we do not claim that this difference is real. We also sliced and diced the data a number of other ways, and the answer was clear: password entry is fine, at least when it comes to user entry errors. Furthermore, we made so many comparisons that the necessary corrections to the $p$-values were unlikely to yield any significant results.

But despite the debate about password masking, it seems that users are simply too good at entering passwords for it to matter whether or not they can see what they are typing, and cannot reject the null hypothesis that masking or unmasking passwords makes no difference to the entry error rate.

\section{Threats to Validity}

This study was carried out as a small academic project, and as such, does not have a large time or financial budget. So while this project is intended to serve as a proof-ofconcept for later work, it is not an exhaustive answer to the questions at hand.

\subsection{Participants}

The first threat is our sampling and number of participants. Our user study used a recruitment agency rather than surveying random students, which was done expressly to 
limit selection bias. While we had a good variety of ages, genders, and smartphone experience, candidates from this agency are often unemployed (which makes sense, given that the study took place on a weekday morning and afternoon). We also excluded UI/UX experts from our selection. Neither of these factors were a problem for the purposes of our work, but it does mean that the selection was not truly random. And even if selection were indeed random, this study is severely underpowered with $n=10$.

It is also important to note only one participant was an English native speaker. While everyone was fluent enough to converse and they could use their keyboard of choice, both the "typical" and "multiword" passwords were based off of english words. This easily could have affected the entry time and error rate-unknown words take longer to visually parse, and are much easier to make mistakes when re-typing due to this lack of familiarity. This also could have made them more difficult to remember, thus hindering the learning response.

\subsection{App}

The app used for the study had some issues. One participant found a bug where pressing the back button would cause the keyboard to flicker on and off for several seconds, preventing the user from entering text. This did not happen very many times, but it still affected the password entry times. The font choice also proved to be problematic. We chose a monospace font with the goal that it would be easily readable, but one participant had considerable trouble with the glyph for the zero digit, and ended up looking for a special symbol that was an exact match to the font choice. Others asked about ambiguous characters, and still others just made their best guess and moved on.

The app was built specifically for this study. Therefore, it was not feasible to place the app directly onto participants' phones. Participants therefore had to use phones that were potentially unfamiliar to them. Typing on an unfamiliar device may lead to both more typing time and errors, and while we hoped to prevent this as much as possible, we could not completely remove the problem.

There is also the problem of the password selection used in the app. The random and multiword passwords used for the study were all generated by 1Password, and the typical passwords were generated by hand. The 1Password password strength metric was used to measure all three password types (to make sure they were roughly equivalent security-wise). However, 1Password is very clear that a strength metric can only test the strength of passwords for which it understands the underlying system that created them [6], meaning it could not accurately assess the security of our typical passwords.

\subsection{Analysis}

With $n=10$, this study is clearly underpowered. Additionally, the number of ways we have looked at the data makes it very difficult to draw interesting conclusions, especially given the small sample size. Even if we did find a few interesting trends, we did so many other comparisons that the necessary corrections to any $p$-values would, in our opinion, render any hypothesis test dubious. 


\section{Conclusions}

After all the debate over the tradeoff between the usability of unmasking passwords and the security of keeping them hidden, it seems that password entry is mostly working as intended. There were slight differences in the error rates of masked passwords and their unmasked counterparts, but this difference was not statistically significant, nor were any of the other ways we sliced and diced the data. People are simply too good at typing passwords for it to matter whether they can see all the characters.

To replicate the research and the figures, download the raw data file (with participant names anonymised) and the $\mathrm{R}$ [16] scripts used to produce the figures from https: //github.com/sten13/password-masking-data.

Acknowledgments. The authors wish to thank Bernhard Tellenbach of the Information Security group and Marc Rennhard of the Institute of Applied Information Systems at the Zurich University of Applied Sciences for funding.

\section{References}

1. Bonneau, J., Preibusch, S.: The password thicket: technical and market failures in human authentication on the web. In: Proceedings of the Workshop of Economics in Information Security 2010 (2010)

2. Chiasson, S., Forget, A., Biddle, R., van Oorschot, P.C.: User interface design affects security: Patterns in click-based graphical passwords. International Journal of Information Security 8(6), 387-398 (Oct 2009). https://doi.org/10.1007/s10207-009-0080-7, http: / /dx . doi.org/10.1007/s10207-009-0080-7

3. Chiasson, S., Oorschot, P.C.V., Biddle, R.: A usability study and critique of two password managers. In: In Proceedings of the 15th USENIX Security Symposium. pp. 1-16 (2006)

4. Enge, E.: Mobile vs desktop usage in 2018: Mobile widens the gap (Apr 2018), https: //www.stonetemple.com/mobile-vs-desktop-usage-study/

5. Gallagher, M.A.: Modeling Password Entry on Mobile Devices: Please Check Your Password and Try Again. Ph.D. thesis, Rice University (May 2015)

6. Goldberg, J.: Toward better master passwords (Aug 2018), https://blog . 1password.com/toward-better-master-passwords/

7. Greene, K.K., Kelsey, J., Frankli, J.M.: Measuring the usability and security of permuted passwords on mobile platforms. Tech. Rep. NISTIR 8040, National Institute of Standards and Technology, Information Access Division, Information Technology Laboratory, 100 Bureau Drive (Mail Stop 8940) Gaithersburg, MD 20899-8940 (Apr 2016)

8. Gruschka, N., Iacono, L.L.: Password visualization beyond password masking. In: Bleimann, U., Dowland, P., Furnell, S., Schneider, O. (eds.) Eighth International Network Conference (INC 2010), Heidelberg, Germany, July 6-8, 2010. Proceedings. pp. 179-188. University of Plymouth (2010), http: / / www. cscan.org/?page=openaccess $\backslash \& i d=111$

9. Gutmann, P.: Engineering Security (Feb 2014), in publication

10. Herley, C., Oorschot, P.C.V.: A research agenda acknowledging the persistence of passwords. IEEE Security \& Privacy 10(2), 28-36 (Jan 2012)

11. Holmes, J.: Stop password masking (Sep 2014), http: / / passwordmasking . com/

12. Melicher, W., Kurilova, D., Segreti, S.M., Kalvani, P., Shay, R., Ur, B., Bauer, L., Christin, N., Cranor, L.F., Mazurek, M.L.: Usability and security of text passwords on mobile devices. In: Proceedings of the 2016 CHI Conference on Human Factors in Computing Systems. pp. 527-539. CHI '16, ACM, New York, NY, USA 
(2016). https://doi.org/10.1145/2858036.2858384, http://doi.acm.org/10.1145/ 2858036.2858384

13. Mitchell, T.: Americans, password management and mobile security (Jan 2017), http://www.pewinternet.org/2017/01/26/ 2-password-management-and-mobile-security

14. Nielsen, J.: The need for web design standards (Sep 2004), https: / / www . nngroup. $\mathrm{com} /$ articles/the-need-for-web-design-standards/

15. Nielsen, J.: Stop password masking (Jun 2009), https://www.nngroup.com/ articles/stop-password-masking/

16. R Core Team: R: A Language and Environment for Statistical Computing. R Foundation for Statistical Computing, Vienna, Austria (2018), https : / /www.R-project .org/

17. Robinson, J.A., Liang, V.W., Chambers, J.A., MacKenzie, C.L.: Computer user verification using login string keystroke dynamics. Trans. Sys. Man Cyber. Part A 28(2), 236-241 (Mar 1998). https://doi.org/10.1109/3468.661150, http://dx.doi .org/10.1109/3468 . 661150

18. Schaub, F., Deyhle, R., Weber, M.: Password entry usability and shoulder surfing susceptibility on different smartphone platforms. In: Proceedings of the 11th International Conference on Mobile and Ubiquitous Multimedia. pp. 13:1-13:10. MUM '12, ACM, New York, NY, USA (2012). https://doi.org/10.1145/2406367.2406384, http://doi . acm. org/10.1145/2406367.2406384

19. Schneier, B.: The pros and cons of password masking (Jul 2009), https://www . schneier.com/blog/archives/2009/07/the\_pros\_and \_co.html

20. Wroblewski, L.: Mobile design details: Hide/show passwords (Nov 2012), https : / / www . lukew.com/ff/entry.asp?1653

21. Yang, Y., Lindqvist, J., Oulasvirta, A.: Text entry method affects password security. In: The LASER Workshop: Learning from Authoritative Security Experiment Results (LASER 2014). USENIX Association, Arlington, VA (2014), https://www.usenix.org/ conference/laser2014/program/agenda/presentation/yang 